\title{
Long-term maintenance of dried acellular matrices
}

Zambon, A. ${ }^{\mathrm{a}, \mathrm{b}}$; Giobbe, G. G..$^{\mathrm{a}, \mathrm{c}}$; Vetralla, M. ${ }^{\mathrm{a}, \mathrm{b}}$; Michelino, F. ${ }^{\mathrm{a}}$; Urbani, L. ${ }^{\mathrm{c}, \mathrm{d}, \mathrm{e}}$; Pantano, M. F.f; Pugno, N. M. ${ }^{\text {f,g,h }}$; De Coppi, Pc ; Elvassore, N. ${ }^{\text {a,b,c }}$; Spilimbergo, S. ${ }^{{ }^{*}}$ ${ }^{a}$ Department of Industrial Engineering, University of Padova, via Marzolo 9, 35131 Padova, Italy bVenetian Institute of Molecular Medicine, Via Orus Giuseppe 2, 35129 Padova, Italy

${ }^{\mathrm{c} S t e m ~ C e l l s ~ a n d ~ R e g e n e r a t i v e ~ M e d i c i n e, ~ G r e a t ~ O r m o n d ~ S t r e e t ~ I n s t i t u t e ~ o f ~ C h i l d ~ H e a l t h, ~ U n i v e r s i t y ~}$ College London, 30 Guilford Street, WC1N 1EH London, United Kingdom

${ }^{\mathrm{d} I n s t i t u t e}$ of Hepatology London, Foundation for Liver Research, 111 Coldharbour Lane, London, SE5 9NT

${ }^{\mathrm{e}}$ Faculty of Life Sciences \& Medicine, King's College London

fLaboratory of Bio-inspired \& Graphene Nanomechanics, Department of Civil, Environmental and Mechanical Engineering, University of Trento, Via Mesiano 77, I-38123 Trento, Italy

${ }^{g}$ Ket Lab, Edoardo Amaldi Foundation, Italian Space Agency, Via del Politecnico snc, 00133 Rome, Italy.

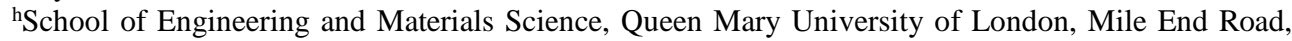
London E1 4NS, UK.

*E-mail of the corresponding author: sara.spilimbergo@unipd.it

\begin{abstract}
Dried and sterile acellular esophageal matrix was obtained within a new drying process based on the use of supercritical carbon dioxide $\left(\mathrm{SC}-\mathrm{CO}_{2}\right)$. Experiments were performed coupling a conventional detergent enzymatic treatment with two different drying methods: (i) $\mathrm{SC}-\mathrm{CO}_{2}$ drying alone; (ii) dehydration in ethanol and a subsequent $\mathrm{SC}-\mathrm{CO}_{2}$ drying. Long term preservation was achieved for several months after drying, demonstrating the maintenance of extracellular matrix (ECM) structure, mechanical properties and biocompatibility within cell repopulation studies in vitro. Overall, the results highlighted the potential of this novel technology to obtain a dry and sterile acellular matrix that can be easily stored for oesophageal regeneration in patients with emergency need.
\end{abstract}

Keywords: decellularized tissue; supercritical drying; carbon dioxide; tissue engineering; long term storage 


\section{Introduction}

Congenital and acquired diseases may involve surgical esophagus substitution. In adult population, esophageal cancer is the eighth most common cancer in the world [1] while esophageal atresia is a congenital anomaly in children that occurs in 1/2500 to 1/4000 live births [2,3]. Esophageal tissue engineering is an emergent alternative for the development of esophageal substitute as repairing technology [4]. Several esophageal engineered substitutes have been explored using synthetic and natural derived scaffolds and materials [5], however, at present, tissue engineering applications to esophageal replacement are limited to enlargement plasties with absorbable non-cellular matrices [6]. Therefore the development and reinforcement of alternative approach are needed to improve the current state of the art. The use of natural matrices in tissue engineering has become extremely promising as tissue substitute for organ reconstruction. Natural acellular matrices are derived from animal or human organs/tissues within a decellularization process which is able to remove cells and immunogenic material [7]. The result is a natural derives scaffold that retains the architecture of the original tissue and also the molecular components of the native extracellular matrix (ECM). Organ decellularization for tissue engineering has become more and more promising for the organ/tissue replacement with preliminary promising clinical experience [8]. Organ decellularization can be successfully applied to esophagus, resulting in a scaffold that maintains ultrastructure and composition of the native extracellular matrix (ECM) [9]. In a clinical scenario, long term preservation and banking of sterile decellularized organs ready for transplantation would be desirable for patients with emergency needs. However, once produced, decellularized scaffolds can't be stored for long time because sterilization and preservation technologies are still lacking. Improvement of the current state of art could be obtained within supercritical carbon dioxide $\left(\mathrm{SC}-\mathrm{CO}_{2}\right)$ drying. $\mathrm{SC}-\mathrm{CO}_{2}$ drying has been already used in for the production of biomaterials such as 3D porous scaffolds [10] and hydrogel [11]. Within $\mathrm{SC}-\mathrm{CO}_{2}$ drying the vapor-liquid interface can be avoided with a minor capillary stress for the product that maintains the original structure. SC- $\mathrm{CO}_{2}$ has a low critical temperature that doesn't degrade thermo sensitive components. $\mathrm{SC}-\mathrm{CO}_{2}$ has also bactericidal properties and it has been deeply investigated as alternative pasteurization and sterilization methods [12,13].

In this scenario the aim of this work is the development of a novel method for the sterilization and long maintenance of decellularized tissue using $\mathrm{SC}-\mathrm{CO}_{2}$ drying.

\section{Materials and Methods}

Porcine esophagus was chosen as test sample. Esophagi were collected from white domestic piglets (Sus scrofa domesticus) from 25 to $40 \mathrm{~kg}$ in weight. All surgical procedures and animal handling were carried out in accordance with UK Home Office guidelines under the Animals (Scientific Procedures) Act 1986 and the local ethics 
committee. Esophageal decellularization was achieved by using a detergent enzymatic treatment (DET) as previously reported [9]. Two different experimental procedures have been explored for the drying: a single step $\mathrm{SC}-\mathrm{CO}_{2}$ drying at $35^{\circ} \mathrm{C}$ and $10 \mathrm{MPa}$ up to 360 min, and a combined double step with ethanol (EtOH) for $80 \mathrm{~min}$ followed by $\mathrm{SC}-\mathrm{CO}_{2}$ drying $\left(\mathrm{EtOH}+\mathrm{SC}-\mathrm{CO}_{2}\right)$ at $35^{\circ} \mathrm{C}$ and $10 \mathrm{MPa}$ up to $90 \mathrm{~min}$. A high pressure sapphire window cell (Separex S.A.S.) was used to carry out the experiments. The internal temperature and pressure were set with a thermostatic bath and an HPLC pump (Gilson 25SC). $\mathrm{CO}_{2}$ (purity $99.990 \%$, Messer) was flowed into the reactor at a constant flow rate of $23 \mathrm{ml} / \mathrm{min}$ until $10 \mathrm{MPa}$; then the pressure was maintained constant by tuning on a micrometric valve. The vessel was depressurized opening the valve at the end of the drying. Samples were packaged for long-term storage in sterile plastic bag at $4^{\circ} \mathrm{C}$ up to 6 months. Samples were weighted after the treatment assuming that all weight loss was due to the moisture removal from the ECM. Weight reduction was expressed as the ratio of $\%$ $\mathrm{W}_{\text {end }} / \mathrm{W}_{\text {start }}$, with $\mathrm{W}_{\text {end }}$ and $\mathrm{W}_{\text {start }}$ expressing respectively the weight of the sample after and before the treatment. Microbiological analysis were performed by means of the standard plate count techniques as previously reported [14] for mesophilic bacterial, mesophilic bacterial spores and yeasts and molds. The enumeration was referred to the weight of initial product and expressed in colony forming units $\mathrm{CFU} / \mathrm{g}$. The degree of inactivation was calculated considering the $\log \left(\mathrm{N} / \mathrm{N}_{0}\right)$, where $\mathrm{N}_{0}(\mathrm{CFU} / \mathrm{g})$ and $\mathrm{N}(\mathrm{CFU} / \mathrm{g})$ is the number of CFU per gram in the untreated and treated sample, respectively. Mechanical characterization was performed with tensile tests with a Midi 10 electromechanical testing machine (Messphysik Materials Testing) at a speed of $0.1 \mathrm{~mm} / \mathrm{s}$ on regular rectangular peaces fixed at the loading frame. Before testing, the dried samples were rehydrated for 24 $\mathrm{h}$ in Phosphate Buffer Saline (PBS-Sigma). Histological analysis were performed on tissue's slice for Haematoxylin and Eosin (H\&E), Masson's trichrome (MT) and Alcian blue (all from Bio-Optica). Cytochemistry analyses on tissue's slices were performed using Alexa Fluor 488 Phalloidin (Thermo Fisher) 1:200 and Hoechst (Sigma Aldrich) $10 \mu \mathrm{M}$ diluted in PBS with $0.1 \%$ Triton X-100 (Sigma Aldrich). Color and fluorescence images were obtained using a color camera mounted on fluorescence inverted microscope (Leica DMI4000). Human bone marrow mesenchymal stem cells (hBMMSCs) were provided by the Cell factory of Policlinico Hospital (Milan, IT) and they were used for scaffold repopulation in vitro. Cell repopulation experiments were performed up to 8 days of culture in customized microwell evaluating the cell adhesion and viability. Cultured samples were analyzed with Live/Dead viability/cytotoxicity kit for mammalian cells (Thermo Fisher) according to manufacturer's instructions

\section{Results and Discussion}

Two methods were investigated for the obtainment of a dried acellular esophagus. A successful dried matrix was reached in a shorter time using the combined ethanol and SC- 
$\mathrm{CO} 2$ treatment. The addition of $\mathrm{EtOH}$ as co-solvent during the process positively influenced the solubility of polar solvent in SC-CO2 [15] and therefore reduced the time of drying. In Fig 1 is represented a cylindrical portion of decellularized esophagus before and after the supercritical drying, and after the rehydration in PBS.

Not dried

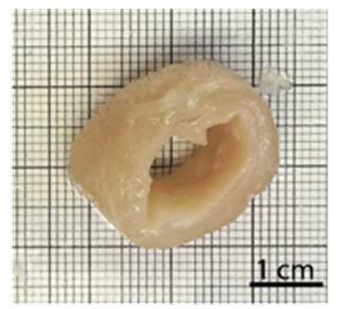

SC-CO2 dried

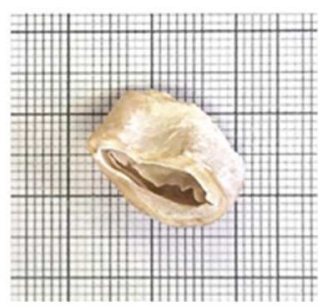

Rehydrated

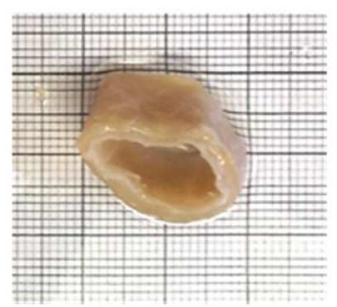

Fig. 1. Picture of cylindrical section of esophagus before (not dried) and after the supercritical drying and the subsequent rehydration in PBS.

Certain shrinkage was observed after the rehydration; mechanical tests didn't show significant differences between the two processes (data not shown) and also a good maintenance of mechanical properties during time (data not shown). The preservation of the esophageal architecture was demonstrated with the presence of the three layers with histological analyses: mucosa, submucosa and muscular layers in all the analyzed samples for H\&E (data not shown). MT staining pointed out the maintenance of the collagen in the lamina propria, submucosa and intermuscular septa of the muscularis externa. AB staining did not show a further decrease of glycosaminoglycan (GAG) after the drying (data not shown). The preservation of the architecture were obtained till 6 months for the H\&E (Fig 2 Top) and confirmed with MT and AB (data not shown). Live and Dead analysis demonstrated good biocompatibility in vitro up to 8 days of culture for the samples dried with the combined process, meaning that $\mathrm{SC}-\mathrm{CO}_{2}$ alone was not able to remove some toxic component from decellularization (data not shown). Repopulation tests demonstrated the presence of live cells on the surface; the repopulation was possible also after 3 and 6 months of storage. Fig 2 (centre and bottom) shows the live and dead analysis at the surface for the total nuclei (Hoechst) in blue and for the dead cells in red (Ethidium). Cells shown high viability in vitro and therefore good biocompatibility even after 8 days of culture in the samples stored for 6 months. The initial load of natural flora population is shown in Table 1. A complete inactivation of the microorganisms was obtained after the EtOH-SC-CO process, while $\mathrm{SC}-\mathrm{CO}_{2}$ drying alone was not able to inactivate completely mesophilic bacterial and spores (data not shown). 

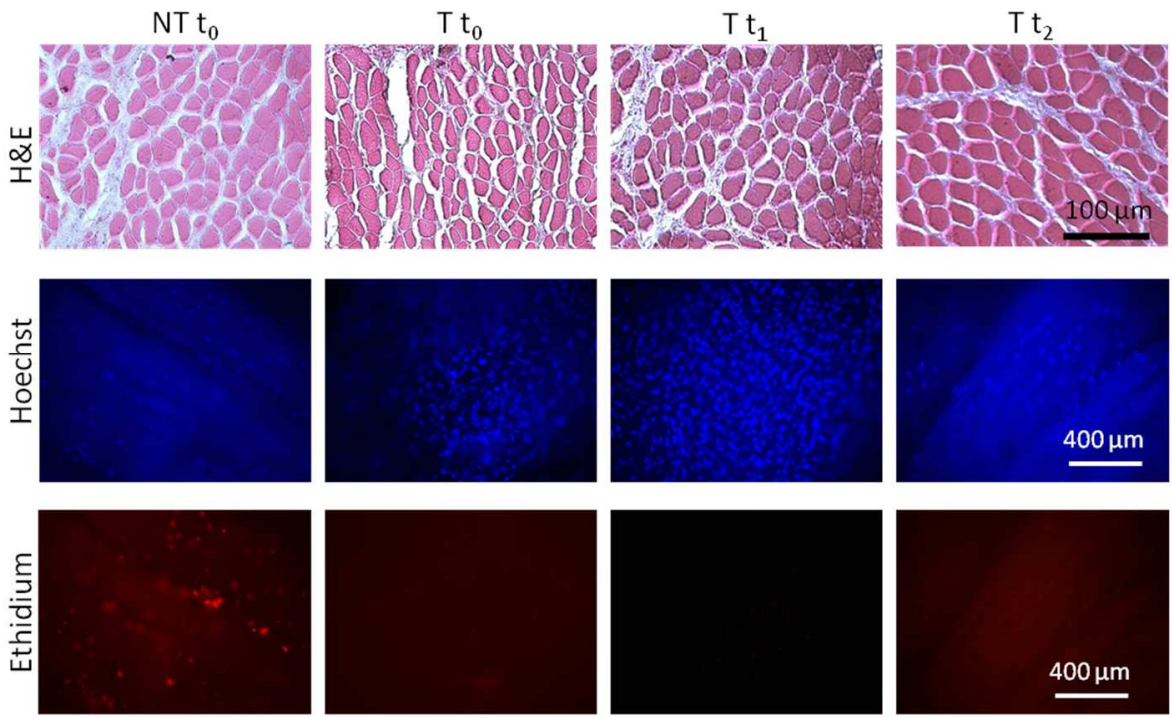

Fig. 2. H\&E staining (Top), live and dead analysis for nuclei with Hoechst (centre) and with Ethidium (bottom) at different storage time.

Table 1. Initial microbiological load of the decellularized esophagus before the treatment

\begin{tabular}{cc}
\hline Microorganism & Log $\mathbf{N}_{\mathbf{0}}[\mathbf{C F U} / \mathbf{g}]$ \\
\hline Mesophilic bacteria & $8.22 \pm 0.89$ \\
Mesophilic spores. & $0.57 \pm 0.12$ \\
Yeast and molds & $8.27 \pm 0.66$ \\
\hline
\end{tabular}

\section{Conclusions}

Overall the results highlighted the potential of this novel method to obtain a dry acellular matrix that can be stored for long term without substantial change of properties from the native tissue. $\mathrm{SC}-\mathrm{CO}_{2}$ drying represents a promising alternative for the long term maintenance of decellularized tissue.

\section{Acknowledgements}

The research leading to these results received funding from Cassa di Risparmio di Trento e Rovereto (CaRiTRo) within the research project "Supercritical decellularization of engineered tissues for clinical application", biomedical science section, 2013. We thanks Lorenza Lazzari for the donation of BM-MSCs from the Cell Factory Bank (Milan-Italy). 


\section{References}

[1] Ferlay, J., et al. "GLOBOCAN 2012 v1. 0, Cancer Incidence and Mortality Worldwide: IARC CancerBase No. 11. Lyon, France: International Agency for Research on Cancer; 2013." Last accessed April (2014).

[2] Spitz L, Kiely E, Pierro A. Gastric transposition in children-a 21-yearexperience. J Pediatr Surg. 2004; 39:276-281.

[3] Sfeir, R., Michaud, L., Salleron, J., \& Gottrand, F. (2013). Epidemiology of esophageal atresia. Diseases of the Esophagus, 26(4), 354-355.

[4] Jensen, Todd, Wael Sayej, and Christine Finck. "Esophageal Tissue Engineering."

Frontiers in Stem Cell and Regenerative Medicine Research: Volume: 33 (2017): 216.

[5] Maghsoudlou P., BSc (Hons.),Eaton S., De Coppi P. Tissue engineering of the esophagus. Seminars in Pediatric Surgery 2014; 23:127-134.

[6] Poghosyan, T., et al. "Esophageal tissue engineering: Current status and perspectives." Journal of visceral surgery 153.1 (2016): 21-29.

[7] Gilbert, Thomas W., Tiffany L. Sellaro, and Stephen F. Badylak. "Decellularization of tissues and organs." Biomaterials 27.19 (2006): 3675-3683.

[8] Elliott, Martin J., et al. "Stem-cell-based, tissue engineered tracheal replacement in a child: a 2-year follow-up study." The Lancet 380.9846 (2012): 994-1000.

[9] Totonelli G, Maghsoudlou P, Georgiades F, Garriboli M, Koshy K, Turmaine M, Ashworth M, Sebire NJ, Pierro A, Eaton S, De Coppi P. Detergent enzymatic treatment for the development of a natural acellular matrix for oesophageal re generation. Pediatric Surgery International, 2013, 29, 87-95.

[10] Pisanti, P., Yeatts, A. B., Cardea, S., Fisher, J. P., \& Reverchon, E. (2012). Tubular perfusion system culture of human mesenchymal stem cells on poly-L-lactic acid scaffolds produced using a supercritical carbon dioxide-assisted process. Journal of Biomedical Materials Research Part A, 100(10), 2563-2572.

[11] Baldino, L., Cardea, S., De Marco, I., \& Reverchon, E. (2015). Complete glutaraldehyde elimination during chitosan hydrogel drying by SC-CO2 processing. The Journal of Supercritical Fluids, 103, 70-76.

[12] Spilimbergo, S., Matthews, M. A., \& Zambon, A. (2018). Supercritical Fluid Pasteurization and Food Safety. In Alternatives to Conventional Food Processing (pp. 153-195).

[13] Qiu, Q. Q., Leamy, P., Brittingham, J., Pomerleau, J., Kabaria, N., \& Connor, J. (2009). Inactivation of bacterial spores and viruses in biological material using supercritical carbon dioxide with sterilant. Journal of Biomedical Materials Research Part B: Applied Biomaterials, 91(2), 572-578.

[14] Ferrentino G, Balzan S, Spilimbergo S. Optimization of supercritical carbon dioxide treatment for the inactivation of the natural microbial flora in cubed cooked ham. Int $\mathbf{J}$ Food Microbiol. 2013 Feb 15;161(3):189-96 
Zambon, A.; Giobbe, G. G.; Vetralla, M.; Michelino, F.; Urbani, L.; Pantano, M. F.; Pugno, N. M.; De Coppi, P; Elvassore, N.; Spilimbergo, $S$.

[15] Ekart, M. P., Bennett, K. L., Ekart, S. M., Gurdial, G. S., Liotta, C. L., \& Eckert, C. A. (1993). Cosolvent interactions in supercritical fluid solutions. AIChE Journal, 39(2), 235-248. 